\title{
Vitamin D reduces hepatic stellate cell proliferation in vitro
}

\author{
P.S. Gibson ${ }^{1}$, K. Hart ${ }^{1}$, E. Fitzpatrick ${ }^{2}$, A. Dhawan ${ }^{2}$, S. A. Lanham-New ${ }^{1}$ and J.B. Moore ${ }^{1,3}$ \\ ${ }^{1}$ Nutritional Sciences Department, Faculty of Health and Medical Sciences, University of Surrey, Guildford GU2 $7 X H$, \\ ${ }^{2}$ Paediatric Liver Centre, King's College Hospital, London SE5 $9 P J$ and ${ }^{3}$ School of Food Science and Nutrition, \\ University of Leeds, Leeds LS2 9JT
}

Activated hepatic stellate cells (HSCs) are a key contributor to liver fibrosis ${ }^{(1)}$ and drive the progression to advanced disease for many liver conditions, including non-alcoholic fatty liver disease. Vitamin D has been shown to have anti-proliferative effects on colorectal cancer cells ${ }^{(2)}$; however less is known about its effects on hepatic stellate cells. The aim of these experiments were to determine in vitro: (i) basal protein expression of the vitamin D receptor (VDR); (ii) confirmation of $1 \alpha, 25(\mathrm{OH})_{2} \mathrm{D}_{3}\left(1,25-\mathrm{VD}_{3}\right)$ phenotypic effect; (iii) the time and dose response to $1,25-\mathrm{VD}_{3}$ treatment.

Three human immortalised cell lines: HT29, colorectal adenocarcinoma; HepG2, hepatocellular carcinoma, and LX-2, hepatic stellate cells, were cultured using standard methods. Several sources of $1,25-\mathrm{VD}_{3}$ were assessed. VDR protein expression was analysed by immunoblotting. VDR and CYP24A1 mRNA expression was measured in LX-2 and HepG2 cells at 0, 1, 2, 4, 6, 8, 10, 12 and 24 hour time points after treatment with $10 \mathrm{nM}$ of $1,25-\mathrm{VD}_{3}$. LX-2, HepG2 and HT29 were treated with a range of $1,25-\mathrm{VD}_{3}$ concentrations $(1 \mu \mathrm{g}-1 \mathrm{ng})$ and cell proliferation was measured by clonogenic assay using crystal violet staining.

Untreated LX-2 cells had a higher abundance of VDR protein than HepG2 cells (Figure 1A). Correspondingly, basal VDR mRNA expression was higher in LX-2 in comparison to HepG2 cells $(P<0.0001)$. However, mRNA expression for CYP24A1 was much lower in LX-2 compared to HepG2 cells $(P<0.0001)$. Treatment with $1,25-\mathrm{VD}_{3}$ dramatically reduced hepatic stellate cell proliferation; a dose-dependent response was observed with $1 \mu \mathrm{M}$ and $100 \mathrm{nM}$ of $1,25-\mathrm{VD}_{3}$ eliciting a significant reduction in cell colonies ( $P=0.0005$ and $P=0.016$ respectively; $n$ 4; Figure 1B) corresponding to $95 \%$ and $82 \%$ fewer colonies. The same trend was seen in HT29 cells $(P=0.053 ; n$; Figure $1 C)$.
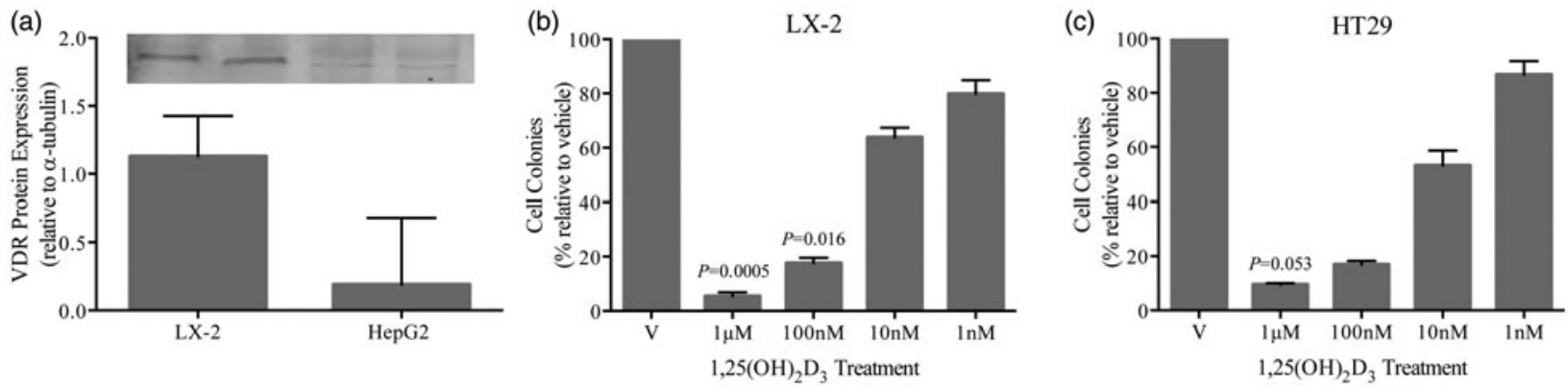

Fig. 1. Basal levels of VDR in LX-2 and HepG2 cells (A). Cell proliferation of LX-2 (B) and HT29 (C) cells in response to $1,25-\mathrm{VD}_{3}$. Data presented as mean + SEM. V: vehicle; VDR: vitamin D receptor.

Unexpectedly, our initial 1,25-VD $\mathrm{VD}_{3}$ treatment appeared to have no effect on VDR and CYP24A1 mRNA expression. After an alternative vitamin D was sourced, a clear reduction in cell proliferation in response to $1,25-\mathrm{VD}_{3}$ was observed in both LX-2 and HT29 cells. Future experiments will determine the associated transcriptional response in co-treatment with lipid loading.

1. Moreira RK (2007) Arch Pathol Lab Med 131: 1728-1734

2. Alvarez-Diaz S, Valle N, Ferrer-Mayorga G et al. (2012) Hum Mol Genet 21 (20): 2157-2165 